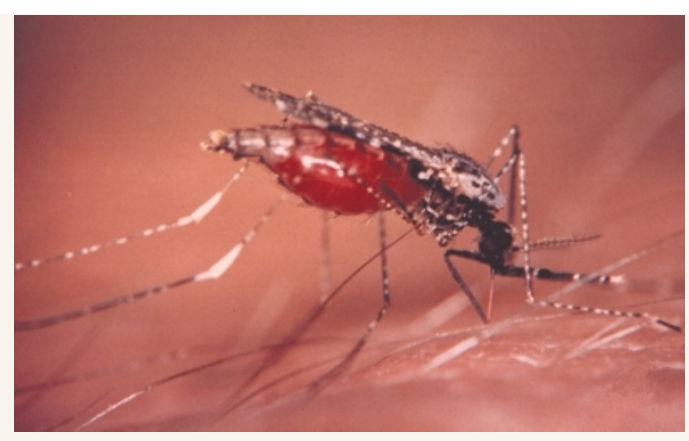

Courtesy of Robert Gwadz.

MALARIA

\section{Resisting drugs}

Chloroquine is a safe and cheap antimalarial drug and it used to be effective. But the malaria parasite, Plasmodium falciparum, has fought back by developing drug resistance. The rising prevalence of chloroquine resistance is increasing still further the health threat posed by malaria. There is an urgent need to understand the basis of chloroquine resistance (CQR), and genetic studies have just opened the door.

Ten years ago, it was shown that CQR segregates as a single Mendelian trait in the P. falciparum genome. The region was narrowed down to $35 \mathrm{~kb}$, but, frustratingly, none of the eight genes identified had mutations that were perfectly associated with CQR. Fidock et al. therefore decided to have a closer look at the region, by searching for small open reading frames (fewer than 100 codons), and used them to screen cDNA libraries. They found a novel gene, $p f c r t$, comprising 13 exons that covered only $3.1 \mathrm{~kb}$, which was predicted to encode a ten-transmembrane channel or transporter protein. The small size of the exons probably explains why the gene was missed in the earlier studies.

Analysis of $p f c r t$ in natural strains of $P$. falciparum revealed several coding variants, and there was perfect association between one amino-acid change in particular - K76 - and resistance. Transfection studies supported the view that $p f c r t$ from resistant parasites could confer resistance on a sensitive strain.

What does pfcrt do? Chloroquine is thought to affect the way the parasite disposes of toxic products from haemoglobin digestion, within a specialized organelle called the digestive vacuole. Fidock et al. have shown that this is where $p f c r t$ is expressed, and that resistant parasites have a significantly reduced $\mathrm{pH}$ within the vacuole. As a membrane protein, the resistant version of PfCRT might antagonize the effects of chloroquine indirectly by altering $\mathrm{pH}$, or directly by affecting the transport of chloroquine across the vacuole membrane. After ten years, the identification of $p f c r t$ is a well-earned prize, which will accelerate progress towards an understanding of drug resistance in malaria.

Mark Patterson

(2) References and links

ORIGINAL RESEARCH PAPER Fidock, D. A. et al. Mutations in the $P$. falciparum digestive vacuole transmembrane protein PfCRT and evidence for their role in chloroquine resistance. Mol. Cell 6, 861-871 (2000) WEB SITES Thomas Wellems's laboratory | The Plasmodium falciparum genome database
H I G H L I G H T S

\title{
New light on night blindness
}

X-linked congenital stationary night blindness (CSNB) is a non-progressive retinal disorder that is characterized by impaired night vision, reduced visual acuity, and frequently, although not always, by nystagmus (uncontrollable eye movement) and myopia. There are two forms of the condition, called complete and incomplete $\mathrm{X}$-linked CSNB, which are distinguishable by measuring the electrophysiological responses of the retina to light. The gene for the incomplete form was identified in 1998, and now, two years on, Nature Genetics reports the cloning of the gene for complete CSNB.

Two research teams tracked down this gene by a mapping and positional-cloning approach. In 22 complete X-linked CSNB families, Bech-Hansen et al. identified 14 different mutations in a novel gene (NYX) that encodes a small leucine-rich proteoglycan called nyctalopin. Meanwhile, Pusch et al. identified NYX deletions in three families with the condition, and went on to identify 14 other mutations in a further 21 families. Both research teams found a spectrum of NYX mutations, including truncations, deletions, point mutations and insertions - BechHansen et al. also found one founder mutation in seven American families with the disease.

Expression studies by the two teams, however, produced different results. BechHansen et al. reported NYX to be expressed robustly in the kidney and retina, whereas Pusch et al. found evidence for a low expression of NYX in several tissues, including the retina, kidney, brain and spinal cord. Pusch et al. suggested that these differences might be due to the use of different PCR templates - this team had used human and fetal tissue, whereas Bech-Hansen et al. had analysed human adult, tissue-specific cDNA libraries. In situ data from Bech-Hansen et al. also showed that NYX transcripts localize to several cell types, including photoreceptor cells, interneurons and ganglion cells, in the different layers of the human retina.

Nyctalopin is a 481 amino-acid protein with many motifs and domains that give clues to its function. It contains a glycosylphosphatidylinositol (GPI)anchor sequence, 11 consecutive leucine- rich repeats (LRRs), an amino-terminal signal peptide, a possible carboxy-terminal cleavage site, and a glycosylation site, among other motifs. Both teams propose that nyctalopin is cleaved at the carboxyl terminus and is GPI-anchored at the extracellular cell membrane as a glycosylated proteoglycan. It is likely that the LRRs confer on nyctalopin the ability to interact with other proteins. LRRs can mediate diverse biological functions, including cell adhesion and migration in Drosophila melanogaster, for example, two LRR proteins, chaoptin and capricious, are cell adhesion molecules that are crucial for fly neuronal development. So, nyctalopin might function as an adhesion molecule that specifies the formation of synapses between rod photoreceptors (which respond to light) and postsynaptic neurons. No doubt, the two teams will now investigate the function of this protein in establishing the complex circuitry of the retina to elucidate how it becomes miswired in patients with complete CSNB.

\section{Jane Alfred}

\section{(2) References and links}

ORIGINAL RESEARCH PAPERS Bech-Hansen N. T.

et al. Mutations in NYX, encoding the leucine-rich proteoglycan nyctalopin, cause X-linked complete congenital stationary night blindness. Nature Genet. 26 , 319-323 (2000) | Pusch, C. M. et al. The complete form of X-linked congenital stationary night blindness is caused by mutations in a gene encoding a leucine-rich protein. Nature Genet. 26, 324-327 (2000)

WEB SITE Congenital stationary night blindness

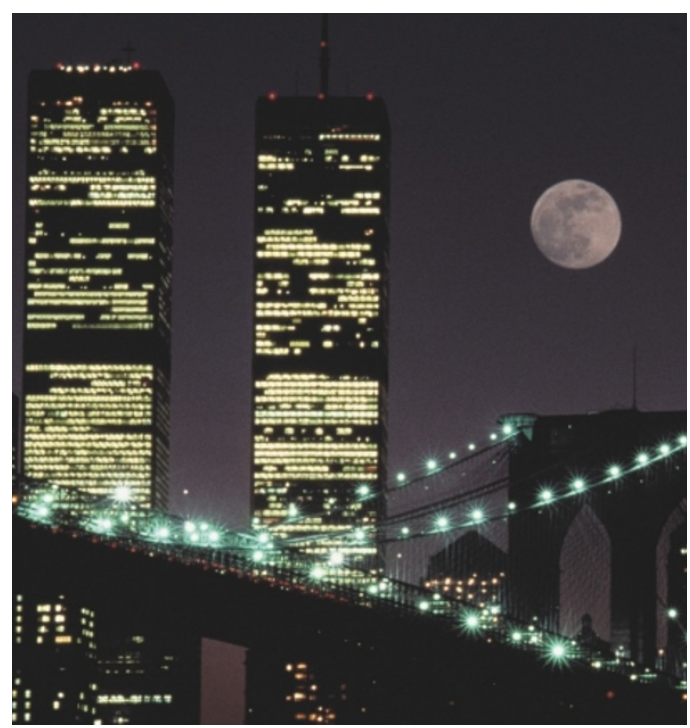

